\title{
Pama-Nyungan grandparent systems change with grandchildren, but not cross-cousin terms or social norms
}

\author{
Catherine Sheard ${ }^{1,2 *}$ (D), Claire Bowern ${ }^{3}$ (D), Rikker Dockum ${ }^{3}$ (D) and Fiona M. Jordan ${ }^{2}$ (D) \\ ${ }^{1}$ School of Earth Sciences, University of Bristol, Bristol BS8 1TQ, UK, ${ }^{2}$ Department of Anthropology and Archaeology, \\ University of Bristol, Bristol BS8 1UU, UK and ${ }^{3}$ Department of Linguistics, Yale University, New Haven. CT 06520, USA \\ ${ }^{\star}$ Corresponding author. E-mail: catherine.sheard@bristol.ac.uk
}

\begin{abstract}
Kinship is a fundamental and universal aspect of the structure of human society. The kinship category of 'grandparents' is socially salient, owing to grandparents' investment in the care of the grandchildren as well as to older generations' control of wealth and cultural knowledge, but the evolutionary dynamics of grandparent terms has yet to be studied in a phylogenetically explicit context. Here, we present the first phylogenetic comparative study of grandparent terms by investigating 134 languages in PamaNyungan, an Australian family of hunter-gatherer languages. We infer that proto-Pama-Nyungan had, with high certainty, four separate terms for grandparents. This state then shifted into either a two-term system that distinguishes the genders of the grandparents or a three-term system that merges the 'parallel' grandparents, which could then transition into a different three-term system that merges the 'cross' grandparents. We find no support for the co-evolution of these systems with either community marriage organisation or post-marital residence. We find some evidence for the correlation of grandparent and grandchild terms, but no support for the correlation of grandparent and cross-cousin terms, suggesting that grandparents and grandchildren potentially form a single lexical category but that the entire kinship system does not necessarily change synchronously.
\end{abstract}

Keywords: Pama-Nyungan; phylogenetic comparative methods; kinship; grandparents; cultural evolution

Media summary: Across 134 Australian languages, Pama-Nyungan systems for naming grandparents shift with grandkids but not social norms.

\section{Introduction}

Kinship is a basic and universal component of the structure of human society (Keesing, 1975; Parkin, 1997) and was one of the foundation concepts in modern anthropology (Morgan, 1871). Kinship organisation forms the backbone of societal rules for inheritance, marriage, alliance and trade (Chapais, 2014; Opie et al., 2014), which in turn affect individuals' reproductive fitness (Chagnon et al., 2017) and shape interactions in daily life (Reiss, 1962). Kinship categories are also clearly lexicalised in the world's languages (e.g. Murdock, 1968; Woodward, 1978; Haspelmath et al., 2001). It is often the case that a single kinship word refers to multiple categories of people, such as the English word 'aunt' meaning both one's father's sister and one's mother's sister. Although the combinatorial possibilities are very large for categorisations that could exist, in practice only a small fraction of possible kinship systems are found cross-culturally (Kemp \& Regier, 2012; Nerlove \& Romney, 1967). Indeed, the comparative study of so-called kinship 'typologies', or common patterns of classifications

\footnotetext{
(c) The Author(s), 2020. Published by Cambridge University Press.. This is an Open Access article, distributed under the terms of the Creative Commons Attribution licence (http://creativecommons.org/licenses/by/4.0/), which permits unrestricted re-use, distribution, and reproduction in any medium, provided the original work is properly cited.
} 
of kin, dates to the nineteenth century, although it is only recently that evolutionary anthropologists have returned to the topic (Jordan, 2011), and the extent to which kinship systems change as a single unit remains largely unknown (Godelier, 2012; Godelier et al., 1998). One outstanding question is the degree to which systems of meaning embedded in language - kinship terminologies - have coevolved with, or are predicted by, patterns of normative social behaviour in ethnolinguistic groups. This tension has characterised kinship studies since Kroeber (1909) and Rivers (1914). Some recent scholars have re-visited these questions with richly nuanced ethnographic and linguistic data (see for example studies in McConvell et al., 2013; and Birchall \& Jordan, 2019), and here we advocate that an evolutionary cross-cultural approach can help to detect potential relationships between cognition, language and culture.

In the nearly 140 years between Morgan and the re-emergence of cross-cultural kinship study, the discipline of cultural phylogenetics has emerged to examine the evolution of cultural and linguistic traits over time (Atkinson \& Gray, 2005; Mace et al., 1994). Although the pseudo-replication caused by related societies' shared common descent has been noted since the 1880s (Tylor, 1889), it was not until the late twentieth century that statistical methods were invented to study the evolution of biological traits along phylogenetic trees (Harvey \& Pagel, 1991), and it was a few years later that these techniques were applied to human language and culture (Blute \& Jordan, 2018; Gray et al., 2007; Mace \& Holden, 2005; Mace et al., 1994). Since then, the study of cultural evolution using phylogenetic comparative methods has been utilised on a wide variety of cultural traits, from the evolution of socio-political hierarchies (Sheehan et al., 2018) to the spread of folktales (da Silva \& Tehrani, 2016). A handful of these studies have focused on kinship systems. Jordan (2011), for example, investigated Austronesian sibling terminologies to show that the relative-age distinction (i.e. older vs. younger sibling) predated distinctions in relative gender (i.e. same-gender sibling vs. opposite-gender sibling). Bantu kinship typologies, which are based on Murdock's (1949) discrete classifications of kinship systems using the number and types of words for cousins, were shown by Guillon and Mace (2016) not to correlate with descent or residence rules, but these rules do correlate with sex biases within the systems (Opie et al., 2014). A recent study found that for a mostly global sample of 936 languages, these Murdock cross-cousin terminologies correlate with community marriage and descent rules (Rácz et al., 2019). To date, however, there has been no cross-cultural phylogenetic study of grandparent terminologies.

Grandparents are an important social category. Grandparents, particularly grandmothers, are a significant source of 'alloparenting', both throughout our evolutionary history (Hrdy, 2011) and in the modern day (Normile, 2017). Researchers have demonstrated a cross-cultural matrilateral bias in the investment of grandparents; across many (but not all) societies, the maternal grandmother, whose genetic relation to the child is certain, provides the most extra-parental help in raising the child (Pashos, 2017; Perry \& Daly, 2017) and can significantly improve grandchild survival and nutritional status (Sear \& Mace, 2008; Sear et al., 2000). The human post-reproductive lifespan, a rarity among mammals, has been posited as an adaptation that specifically allowed grandmothers to help raise their grandchildren in what is known as the 'grandmother hypothesis' (Hawkes et al., 1998; K. Hill \& Hurtado, 1991). Grandparents play a variety of significant cultural and social roles within a society, as older individuals tend to control a considerable amount of wealth and skills (Møllegaard \& Jæger, 2015). Moreover, specific terms that record further details about the social category of the grandparent - for example, that a person is a grandmother rather than a grandfather or a paternal grandmother rather than maternal one - can aid in the categorisation of one's kin by socially salient categories, such as keeping track of moieties, noting lines of inheritance, or working out preferred marriage partners (Chapais, 2014).

Across the world's languages, classification systems for grandparents vary. Many dialects of English have just two terms for grandparents, 'grandmother' and 'grandfather', which distinguish gender but not lineage (i.e. paternal vs. maternal grandparents); in contrast the Australian language Alyawarr has four unique terms for each role (Yallop, 1977), while the Hawaiian words tutu or $k u k u$ can refer to any grandparent. As with the more famous kinship systems based on cousin terms (Goody, 1970; Guillon \& 
Mace, 2016; Rácz et al., 2019), cross-cultural variation in grandparent systems is thought to vary with aspects of social structure, such as community marriage organisation (Dole, 1969; McConvell \& Dousset, 2012; Parkin, 2012) and post-marital residence (McConvell, 2012), although the extent to which grandparent terms change in concert with other categories of kinship is unknown (Dzibel \& Dziebel, 2007; Read, 2013). Ethnolinguistic groups do not, however, represent independent samples. Vertical transmission between historically related societies often shapes extant patterns, necessitating phylogenetic models in the study of cross-cultural variation (Gray et al., 2007; Mace et al., 1994). Horizontal transmission, or borrowing between cultures in contact, can also determine traits' evolutionary trajectories (Currie et al., 2010; Nunn et al., 2006). A study of the relationship between social practices and grandparent systems (or indeed any kinship classification) would therefore need to account for the potential of both historical and spatial autocorrelation. As a well-resolved global language phylogeny is currently lacking, and given the current body of evidence suggesting the substantial role local processes can play in cultural macroevolution families (Dunn et al., 2011; Fortunato \& Jordan, 2010; Moravec et al., 2018; Surowiec et al., 2019), this limits a study of the variation in grandparental systems to the scale of a language family.

Pama-Nyungan is the world's largest hunter-gather language family, comprising over 300 languages traditionally spoken across a region encompassing over $90 \%$ of the Australian continent (Bowern \& Atkinson, 2012). Although Aboriginal Australians have inhabited Australia for over 50,000 years (Malaspinas et al., 2016), Pama-Nyungan probably originated between 4,500 and 7,000 years ago in the Gulf Plains region and appears to have quickly spread together with cultural innovations such as agricultural intensifications and rock art (Bouckaert et al., 2018). During this expansion, there is ambiguous evidence for genetic replacement, suggesting that these languages spread through assimilation rather than comprehensive takeovers (Bouckaert et al., 2018). The approximately 100 non-Pama-Nyungan languages found in Australia are all restricted to the north coast; beyond divisions into 27 linguistic families, the phylogenetic relationships among these groups remain opaque (McConvell \& Bowern, 2011). Within the domain of kinship specifically, Australian societies are notable for social categories such as moieties, skins and sections (Lacrampe et al., 2018; Scheffler, 1978). Generational moieties, such as those found in the Western Desert languages, classify grandparents and grandchildren in the same moiety (White, 1981); in these languages, grandparent and grandchild terms are frequently colexified. Some Pama-Nyungan languages, such as Yidiny and Dyirbal, also have kinship avoidance registers, where sons-in-law and mothers-in-law observe strict behavioural and linguistic taboos in one another's presence, such as using a separate avoidance vocabulary (Dixon, 1990). Linguistic exogamy is also common in Australia (Clendon, 2006; J. H. Hill, 1978), which has been demonstrated to affect borrowing rates generally (Bowern et al., 2011) and kinship term borrowing specifically (Bowern, 2010; Haspelmath, 2008).

The large sample size of Pama-Nyungan languages within a single well-resolved phylogeny (Bouckaert et al., 2018; Bowern \& Atkinson, 2012), coupled with a continent-level radiation of languages, permits the use of phylogenetic comparative methods to investigate the co-evolution of language and culture across space and time. By focusing explicitly on a group of hunter-gatherer languages, we are able to model the dynamics of language change without the potential confounding effects of the many cultural and political changes that accompanied domestication and agricultural transitions (Creanza et al., 2017). Farming practices date to approximately 11,000 years ago (Bramanti et al., 2009), whereas the Upper Palaeolithic transition, characterised by the sudden appearance of technological and cultural complexity in early modern humans, was approximately 45,000 years ago (Powell et al., 2009); comparative studies of wholly agricultural populations therefore may not be representative of the entirety of human evolution (Bowern et al., 2011). Moreover, even if there is no difference in the evolutionary dynamics of hunter-gatherer and of agriculturalist kinship systems, the unique biogeographic and anthropological history of each region is expected to separately shape different language families (Dunn et al., 2011; Fortunato \& Jordan, 2010; Moravec et al., 2018). Phylogenetic comparative analyses of aspects 
of kinship systems have been performed on Indo-European (Fortunato \& Jordan, 2010), Bantu (Guillon \& Mace, 2016; Opie et al., 2014) and Austronesian languages (Fortunato \& Jordan, 2010; Jordan, 2011), as well as a small number of cross-family studies (Boden et al., 2014; Moravec et al., 2018; Rácz et al., 2019; Walker \& Bailey, 2014), but Australian studies have been largely absent from this literature.

Here we use Bayesian phylogenetic comparative analyses to reconstruct the sequence of changes in Pama-Nyungan grandparent term systems and to test the role of social structure in shaping these changes. Specifically, we evaluate the effects of phylogenetic signal (vertical transmission), spatial signal (horizontal transmission), community marriage organisation (specifically, the effect of linguistic exogamy) and post-marital residence. Finally, because variation in kinship terminologies is often collapsed into a small number of typologies (Dzibel \& Dziebel, 2007; Fox, 2008; Murdock, 1949; Read, 2013) - typically based on cousin terminology, and often applied without validating that these classifications are meaningful in the sample under study - we test for kinship system synchrony within our sample. We thus compare changes in grandparent systems with (1) changes in grandchild term systems and (2) changes in terms for cross-cousins.

\section{Methods}

\section{Data collection}

Terms for grandparents were obtained from the Australian linguistics database CHIRILA (Bowern, 2016) for 134 Pama-Nyungan languages. Here 'grandparents' are defined as the linguistic terms for a speaker's mother's mother (MM), mother's father (MF), father's mother (FM) and father's father (FF); definitions of common abbreviations and terms can be found in Table 1. These terms were then categorised based on which grandparent terms, if any, were merged; in Pama-Nyungan, the relevant distinctions are between the two parallel grandparents ( $\mathrm{MM}$ and $\mathrm{FF}$ ), the two cross grandparents (MF and FM), the two female grandparents (MM and FM) and the two male grandparents (FF and MF). These scores formed the basis of a discrete post-hoc categorisation into 'systems' (Table 2).

For 58 of these languages, we were able to obtain further data on the terms for 'grandchild' (56 languages) and/or 'cross-cousin' (51 languages). Most of this data came from the Australian language database CHIRILA (Bowern 2016) and the social organisation and kinship database Austkin (Dousset et al. 2010); full citations for each language are provided in the supplementary material.

As with grandparents, there are four core grandchild categories: daughter's daughter (DD), daughter's son (DS), son's daughter (SD) and son's son (SS). In Pama-Nyungan languages, it is common for grandchild and grandparent terms to be colexified; we thus noted if any grandchild term was the same as any grandparent term. Because grandchildren terms tended to be complicated, we devised a posthoc coding scheme based on four binary variables: (1) whether any of the grandchild terms was a generic (a term for which $\mathrm{DD}=\mathrm{DS}=\mathrm{SD}=\mathrm{SS}$ ); (2) whether any of the terms were merged by gender of the referent ( $\mathrm{DD}=\mathrm{SD}$ and/or SS = DS); (3) whether any of the terms were merged by the parent of the referent ( $\mathrm{DD}=\mathrm{DS}$ and/or SS = SD); and (4) whether each grandchild could be assigned a unique term.

Cross-cousin terms were coded according to a similar set of four criteria: (1) whether there was a generic term that could be applied to all four cross-cousins; (2) whether any terms were merged by gender of the referent $(\mathrm{MBD}=\mathrm{FZD}$ and/or $\mathrm{MBS}=\mathrm{FZS})$; (3) whether any terms were merged by the parent of the referent $(\mathrm{MBD}=\mathrm{MBS}$ and/or FZD = FZS); and (4) whether each cousin could be assigned a unique term.

\section{Ancestral dynamics of grandparents}

All phylogenetic analyses were performed on a distribution of 100 trees from Bouckaert et al. (2018), which contained tips for 110 of the 134 languages surveyed. As many comparative analyses require 
Table 1. Glossary of kinship terms and abbreviations. These terms are not exhaustive and do not encompass the diversity of human experience (e.g. step-grandparents, same-gender marriages, non-binary genders, differentiation between sex and gender). By convention, kinship terms are typically abbreviated such that WXY is interpreted as W's X's Y. M mother, $\mathrm{F}=$ father, $\mathrm{D}=$ daughter, $\mathrm{S}=$ son, $\mathrm{Z}=$ sister and $\mathrm{B}=$ brother

\begin{tabular}{ll}
\hline MM & Speaker's mother's mother; maternal grandmother \\
\hline MF & Speaker's mother's father; maternal grandfather \\
\hline FM & Speaker's father's mother; paternal grandmother \\
\hline FF & Speaker's father's father; paternal grandfather \\
\hline Cross grandparents & Opposite-gender parents of speaker's parents; MF and FM \\
\hline Parallel grandparents & Same-gender parents of speaker's parents; MM and FF \\
\hline $\begin{array}{c}\text { Community marriage } \\
\text { organisation }\end{array}$ & $\begin{array}{c}\text { General community norms toward marriage within or between groups. Here we focus } \\
\text { on specifically linguistic exogamy, or marriage outside of an ethnolinguistic group. } \\
\text { Endogamy: marriage within the (here, linguistic) group. Agamy: no tendency to } \\
\text { either endogamy or exogamy. See Kirby et al. (2016) and Murdock (1967) for more } \\
\text { information }\end{array}$ \\
\hline $\begin{array}{c}\text { Post-marital residence } \\
\text { The prevailing pattern of residence of a couple after marriage. Patrilocal: newly } \\
\text { married couple typically resides near the husband's family. Matrilocal: newly } \\
\text { married couple typically resides near the wife's family. See Kirby et al. (2016), } \\
\text { Moravec et al. (2018) or Murdock (1967) for more information }\end{array}$ \\
\hline
\end{tabular}

ultrametric trees, the tips of all recently extinct languages were extended to the present day. To assess the phylogenetic signal of the grandparent systems, we used a $\lambda$-transformation using the command 'fitDiscrete' in the R package geiger (Harmon et al., 2008). The ancestral grandparent system was calculated for each tree in a maximum likelihood framework using the command 'ace' in the R package ape (Paradis et al., 2004); the median values across the 100 trees are reported here, and the results computed on a consensus tree from this distribution are shown in Figure 2. The Bayesian analysis to reconstruct the dynamics of grandparent system transitions also produced an estimation of the root ancestral state (see below); we report these additional results for completeness. Further details on these analyses can be found in the supplementary material.

To assess the potential for spatial signal - which could be a result of horizontal transmission and/or an indication that grandparent systems are partially driven by local environmental variables - we obtained latitude and longitude coordinates of point estimate locations for 130 of the 134 languages from Glottolog (Hammarström et al., 2018), with coordinates from the remaining four languages provided by author $\mathrm{CB}$. We then performed a Mantel test using the command 'mantel.rtest' from the R package ade4 (Dray \& Dufour, 2007), which tests for correlation between two matrices, with 9,999 permutations. The distance matrix was taken as straight-line distances between each language's point estimate; the grandparent system 'distance' was set to be 0 if languages shared a system and 1 otherwise.

To assess the internal dynamics of grandparent systems, we used the command 'Multistate' in BayesTraits (Pagel et al., 2004), which calculates the transition rate between systems. To minimise the number of parameters to be estimated with this relatively small sample size, we limited our analysis to the four most common grandparent systems - (1) four separate terms, (2) merging the genders, (3) merging the parallel grandparents and (4) merging the cross grandparents (see Table 2) - and employed a Markov chain Monte Carlo (MCMC) reversible jump process, which allows parameters to be set to 0 . All priors were set to be drawn from an exponential distribution with a mean of 10 , and for each tree the chain was run for 1,010,000 iterations with a burn-in of 10,000 and a sampling rate of 1,000 . We report the median values of each parameter across all tree topologies in the distribution. This analysis also provides a Bayesian framework in which to estimate the root ancestral state, in a model allowing transitions between the four most common systems to vary asymmetrically; these results are presented alongside the maximum-likelihood ancestral state estimates (see above). 


\section{Co-evolution between grandparent system and cultural norms}

We obtained information on the community marriage organisation for 29 societies from D-PLACE (Kirby et al., 2016), which for Australian data is based on Binford (2001)'s cross-cultural survey of hunter-gatherer societies. D-PLACE scores 'community marriage organisation' using five categories, four of which were found in our sample. We collapsed this to three categories: exogamous, agamous and endogamous (D-PLACE 'endogamous deemed' and 'endogamous segmented'). Data for a further 46 languages were obtained from the ethnographic literature; a list of sources and scores is available in the supplementary material. We also obtained information on community post-marital residence norms for 50 languages from Moravec et al. (2018). All languages in our sample are either patrilocal (newly married couples typically reside near the husband's family) or matrilocal (newly marriage couples typically reside near the wife's family).

To assess the relationship between community marriage rules and grandparent system, we ran binary Bayesian phylogenetic mixed models (BBPMM) using the R package $M C M C g l m m$ (Hadfield, 2010) across a sample of 100 trees; full details of model parameters can be found in the supplementary material.

To ensure model convergence when testing the relationship between grandparent systems and community marriage rules, we restricted our sample to only the two most common grandparent systems (four separate terms and merging by gender). Because we were specifically interested in the effect of linguistic exogamy on grandparental systems, we also merged endogamy and agamy into a single category for the analyses reported here. (A set of analyses with agamy and exogamy instead merged is reported in the supplementary material.) Owing to the long history of presenting cross-cultural data without a phylogenetic correction (for recent examples, see Rácz et al., 2019; Schulz et al., 2019; or Whitehouse et al. 2019), we also assessed the relationship between grandparent terminologies and marriage systems in a non-phylogenetic framework, the results of which we report in the supplementary material.

To further investigate the precise drivers behind this apparent relationship between grandparent systems and social norms, and in an attempt to increase our sample size, we also tested the relationship between both community marriage organisation and post-marital residence with each of the four main features of Pama-Nyungan grandparent systems (and the basis of our original coding scheme): merging the female grandparents $(\mathrm{MM}=\mathrm{FM})$, merging the male grandparents $(\mathrm{FF}=\mathrm{MF})$, merging the cross-grandparents $(\mathrm{MF}=\mathrm{FM})$ and merging the parallel-grandparents $(\mathrm{MM}=\mathrm{FF})$.

\section{Co-evolution with grandchild and cross-cousin systems}

Finally, we assessed the internal synchronicity of the Pama-Nyungan kinship system by evaluating the co-evolution of grandparent systems with two other other kinship categories, grandchild and crosscousin systems. We first considered whether polysemy between grandparent and grandchild terms is related to grandparent systems; the phylogenetic results are presented here, with non-phylogenetic results presented in the supplementary material. We then ran BBPMMs comparing grandparent systems and each of three traits of grandchild and cross-cousin systems: (a) whether a single generic word can be applied to all members of the category; (b) whether the system merges terms by gender of the referent; and (c) whether the system merges terms by the parent of the referent.

\section{Results}

\section{Grandparent systems: an overview}

The most common system, found in 86 languages, had four separate terms for all four kinship roles (here abbreviated AS). The second-most common system, found in 23 languages, merged the gender of the referent $(\mathrm{MM}=\mathrm{FM}$ and $\mathrm{FF}=\mathrm{MF}$, as in English, abbreviated $\mathrm{MG})$. The next most common 
system, found in 13 languages, had a single term for the so-called 'parallel' grandparent, the parent's same-gender parent $(\mathrm{MM}=\mathrm{FF}$, abbreviated MP). The four remaining systems are described in Table 2 .

Grandparent systems showed a strong phylogenetic signal $\left(\mathrm{AIC}_{\lambda}=286.7, \mathrm{AIC}_{\lambda=1}=286.7, \mathrm{AIC}_{\lambda=0}=\right.$ 348.8) but showed no geographic signal (Mantel test, $P=0.417$ ) (Figure 1); geographic distance was thus disregarded as a potential confounding variable in all further analyses.

\section{Ancestral dynamics of grandparents}

With very high probability, the ancestral grandparent system of all Pama-Nyungan languages was AS, four separate terms ( $P=0.9996$ assuming equal rates, $P=0.9991$ assuming symmetric rates) (Figure 2 ). This remained true even when rare systems were excluded from the analysis $(P=0.9993$ assuming equal rates; $P=0.9990$ assuming symmetric rates; or $P=0.9989$ allowing asymmetric rates in a Bayesian framework). For the dynamics reconstructed on the consensus tree, there were at least nine independent instances of merging grandparents by gender (MG), eight independent instances of merging parallel grandparents (MP) and four independent instances of merging cross grandparents (MC). Although a large number of independent cultural innovations can be indicative of high rates of horizontal transmission, given the lack of geographic signal and the high phylogenetic fidelity, these transitions are more likely to be the products of a flexible trait.

Among the four most common grandparent systems, the 'Multistate' analysis reconstructed a high rate of transition between the ancestral four-term AS system and a three-term MP system $(\mathrm{MM}=\mathrm{FF})$ (median instantaneous transition rate $\mathrm{q}=41$ ), with some transitions also reconstructed from the fourterm system to a two-term MS system $(\mathrm{MM}=\mathrm{FM}$ and $\mathrm{MF}=\mathrm{FF})(q=4)$ (Figure 3$)$. Both of these states would then with high probability undergo a subsequent transition to the three-term MC system (MF $=\mathrm{FM})\left(q_{\mathrm{MP} \rightarrow \mathrm{MC}}=37\right.$ and $\left.q_{\mathrm{MS} \rightarrow \mathrm{MC}}=39\right)$, with additional transitions from MP to MS $(q=33)$. We observed on average no transitions between the tertiary state (MC) and the primary state (AS) $\left(q_{\mathrm{AS} \rightarrow \mathrm{MC}}=0,95 \%\right.$ credible interval $0-27 ; q_{\mathrm{MC} \rightarrow \mathrm{AS}}=0,95 \%$ credible interval $\left.0-106\right)$, nor any transitions from secondary state MS to secondary state MP $(q=0,95 \%$ credible interval $0-58)$. Although it might be reasonable to assume that some of these shifts are the result of contact, particularly in multilingual and/or exogamous communities where parents and grandparents may be from different language backgrounds, we found no overall evidence of spatial structuring within Pama-Nyungan languages.

\section{Co-evolution with community marriage organisation}

Of the 75 languages for which we were able to obtain data on community marriage rules, one (Warumungu) had a unique grandparent system and was therefore omitted, leaving a total of 74 languages across four systems. We expected to find that societies with more potential resources for term innovations - i.e. those with linguistic exogamy, vs. linguistic endogamy - would have a greater number of terms. We found, however, no effect of community marriage organisation on grandparental systems ( $\mathrm{pMCMC}=0.928$, Figure 4). Similarly, we found no evidence of a relationship between linguistic exogamy and any individual component of grandparental systems across all 75 languages, namely merging the grandmothers $(\mathrm{MM}=\mathrm{FM}, \mathrm{pMCMC}=0.802)$, merging the grandfathers $(\mathrm{MF}=\mathrm{FF}$, $\mathrm{pMCMC}=0.836)$, merging the cross grandparents $(\mathrm{MF}=\mathrm{FM}, \mathrm{pMCMC}=0.162)$ or merging the parallel grandparents $(\mathrm{MM}=\mathrm{FM}, \mathrm{pMCMC}=0.918)$.

\section{Co-evolution with post-marital residence}

Of the 50 languages for which we were able to obtain data on post-marital residence norms, four had grandparent systems found in only one or two languages and were thus omitted, leaving a sample of 46. Owing to the differential resource allocation in grandchildren between maternal and paternal grandparents (Pashos, 2017), as well as long-standing beliefs that post-marital residence shapes 
Table 2. Pama-Nyungan grandparent systems. Languages were first coded by whether they had the same words for female grandparents $(M M=F M)$, male grandparents $(M F=F F)$, parallel grandparents $(M M=F F)$ and cross grandparents $(\mathrm{MF}=\mathrm{FM})$, and then classified into discrete systems

\begin{tabular}{|c|c|c|c|c|c|}
\hline Description & $\begin{array}{l}\text { Abbreviation } \\
\text { used in-text }\end{array}$ & $\begin{array}{l}\text { Kinship } \\
\text { equivalences }\end{array}$ & $\begin{array}{l}\text { Number of } \\
\text { languages }\end{array}$ & $\begin{array}{l}\text { Percentage } \\
\text { of languages }\end{array}$ & $\begin{array}{l}\text { Example } \\
\text { language }\end{array}$ \\
\hline All separate terms & AS & none & 86 & $64 \%$ & Alyawarr \\
\hline Merge by gender & MG & $\begin{array}{l}\mathrm{MM}=\mathrm{FM}, \\
\mathrm{FF}=\mathrm{MF}\end{array}$ & 23 & $17 \%$ & Pitjantjatjara \\
\hline $\begin{array}{l}\text { Merge 'parallel' } \\
\text { grandparents }\end{array}$ & MP & $\mathrm{MM}=\mathrm{FF}$ & 13 & $10 \%$ & Djapu \\
\hline $\begin{array}{l}\text { Merge 'cross' } \\
\text { grandparents }\end{array}$ & MC & $M F=F M$ & 6 & $4 \%$ & Yagara \\
\hline $\begin{array}{l}\text { Merge cross and } \\
\text { parallel }\end{array}$ & - & $\begin{array}{l}M F=F M, \\
M M=F F\end{array}$ & 4 & $3 \%$ & Kuku Yalanji \\
\hline $\begin{array}{l}\text { Merge by gender of } \\
\text { parent }\end{array}$ & - & $\begin{array}{l}\mathrm{MM}=\mathrm{MF} \\
\mathrm{FM}=\mathrm{FF}\end{array}$ & 1 & $<1 \%$ & Nyawaygi \\
\hline $\begin{array}{l}\text { Merge } \\
\quad \text { grandmothers }\end{array}$ & - & $\mathrm{MM}=\mathrm{FM}$ & 1 & $<1 \%$ & Wemba Wemba \\
\hline
\end{tabular}

Figure 1. Distribution of Pama-Nyungan grandparent systems across space. Each system is plotted at the society's centroid. The three least common systems (six languages) are omitted.

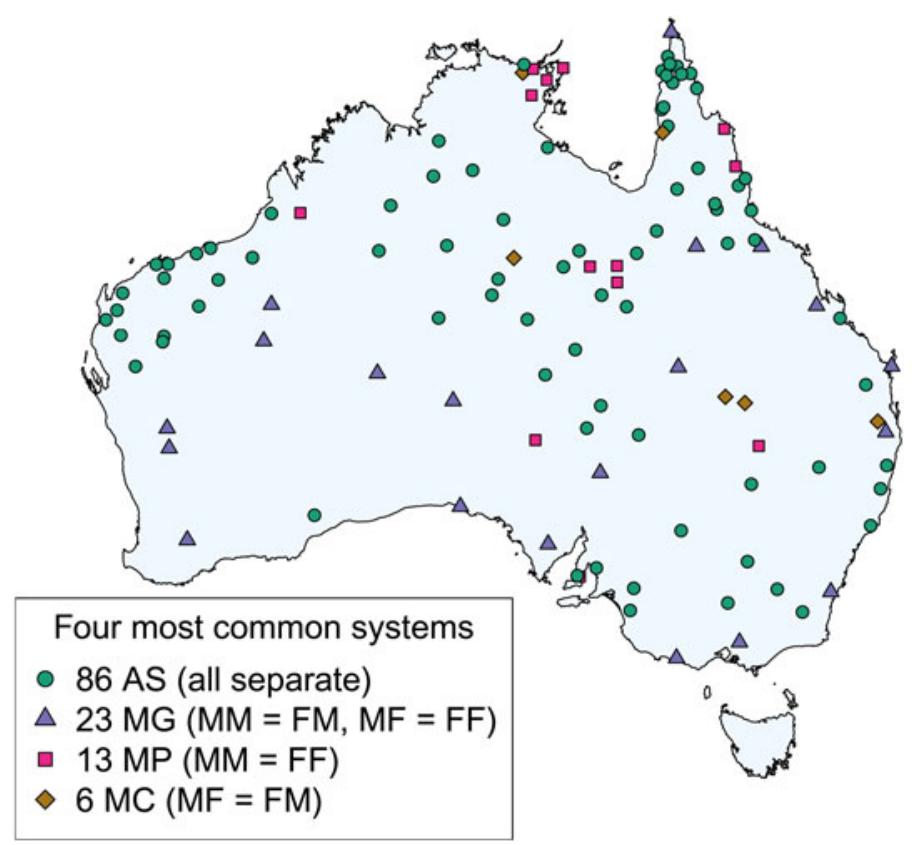

kinship systems more generally (Murdock, 1949), we expected to see differences in the types of grandparental systems employed by matrilocal and patrilocal societies. We observed, however, no such effect (Figure 4, pMCMC $=0.730$ ).

As with community marriage organisation, we also found no evidence that post-marital residence correlates with any particular aspect of grandparent systems across all 50 languages, namely merging the grandmothers $(\mathrm{MM}=\mathrm{FM}, \mathrm{pMCMC}=0.998)$, merging the grandfathers $(\mathrm{MF}=\mathrm{FF}, \mathrm{pMCMC}=$ $0.990)$, merging the cross grandparents $(\mathrm{MF}=\mathrm{FM}, \mathrm{pMCMC}=0.600)$ or merging the parallel grandparents $(\mathrm{MM}=\mathrm{FM}, \mathrm{pMCMC}=0.496)$. 


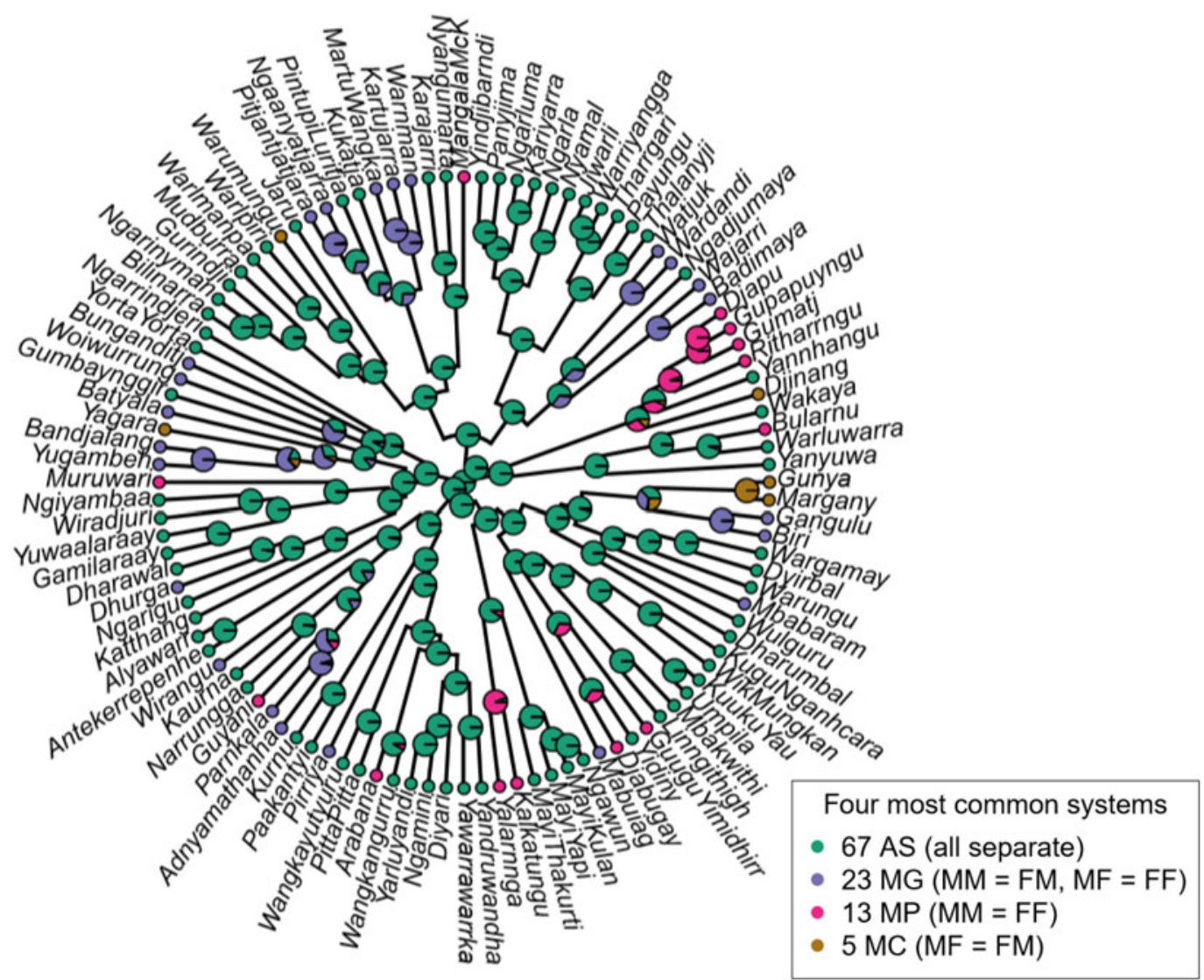

Figure 2. Distribution of Pama-Nyungan grandparent systems across time. The least common systems (two languages) are omitted. For the purposes of display, the topology shown is a consensus tree from the posterior distribution in Bouckaert et al. (2018), and ancestral states assume equal transition rates between all states; the results presented in-text are the median values across the 100 tree topologies.

\section{Co-evolution with grandchild and cross-cousin systems}

Finally, to test how well Pama-Nyungan grandparent terms fit into the concept of 'kinship' as a single synchronous typology, we evaluated the relationship between grandparent systems and grandchildren or cross-cousin terms. Although grandchild and grandparent systems may appear to be correlated, these relationships largely do not survive phylogenetic correction. For example, of the 56 languages for which we were able to obtain grandchild data, 43 languages (77\%) have a word that can be applied to both a grandparent and a grandchild category. These grandparent-grandchild reciprocals are common in systems that merge the gender of the grandparent (100\%) and in systems with separate terms for each grandparent (85\%); it is rarer in systems that merge the parallel (50\%) or cross (40\%) grandparents. This association between grandparent systems and grandparent-grandchild polysemy, however, was indistinguishable from random variation within a phylogenetic framework ( $\mathrm{pMCMC}=0.736$ ).

Similarly, $\mathrm{MG}$ languages $(\mathrm{MM}=\mathrm{FM}$ and $\mathrm{FF}=\mathrm{MF})$ appear likely to merge the grandchildren terms by the gender of the referent ( $\mathrm{SS}=\mathrm{DS}$ and/or $\mathrm{DD}=\mathrm{SD}$ ) and less likely to merge the grandchildren terms by identity of the parent $(\mathrm{SS}=\mathrm{SD}$ and/or $\mathrm{DD}=\mathrm{DS})$, but these relationships did not hold after phylogenetic correction ( $\mathrm{pMCMC}=0.408$ and $\mathrm{pMCMC}=0.288$ respectively). MP languages $(\mathrm{MM}=\mathrm{FF})$, however, were indeed most likely to have a generic word for 'grandchild', independent of shared cultural history $(\mathrm{pMCMC}<0.001)$. 


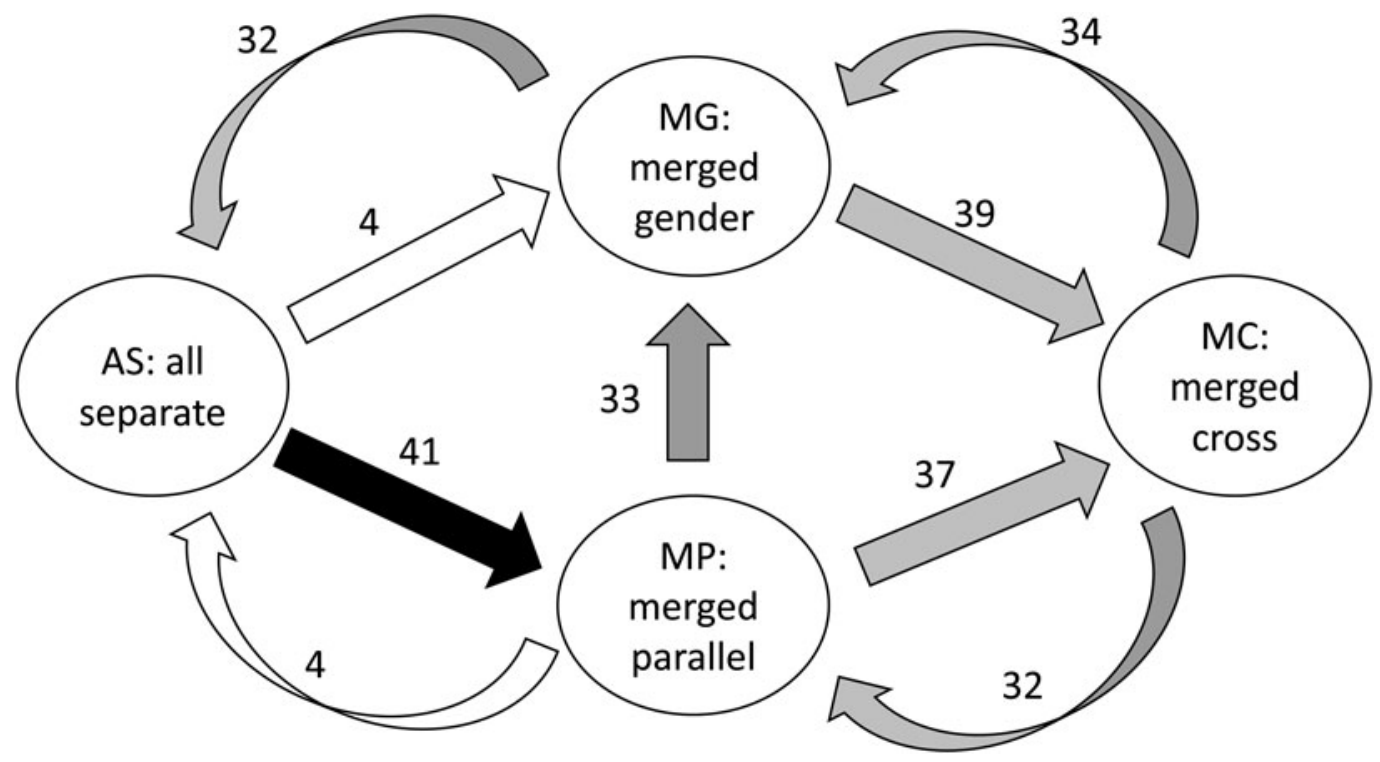

Figure 3. Evolutionary transitions between grandparental systems. The ancestral state has four separate terms for the four grandparents (AS); this state transitions to either a three-term system with merged parallel grandparents (MM = FF, MP), or with an order of magnitude smaller probability, to a two-term system with merged genders ( $M M=F M$ and $M F=F F, M G)$. These secondary states could then subsequently transition to a three-term state with the cross grandparents merged ( $F M=M F, M C)$. Arrow colour indicates the modelled transition rate, $q$, over an infinitely small time period; see Pagel (1994) for more details. An absent arrow (e.g. from MS to MP or between AS and MC) indicates that this transition rate was estimated as 0.
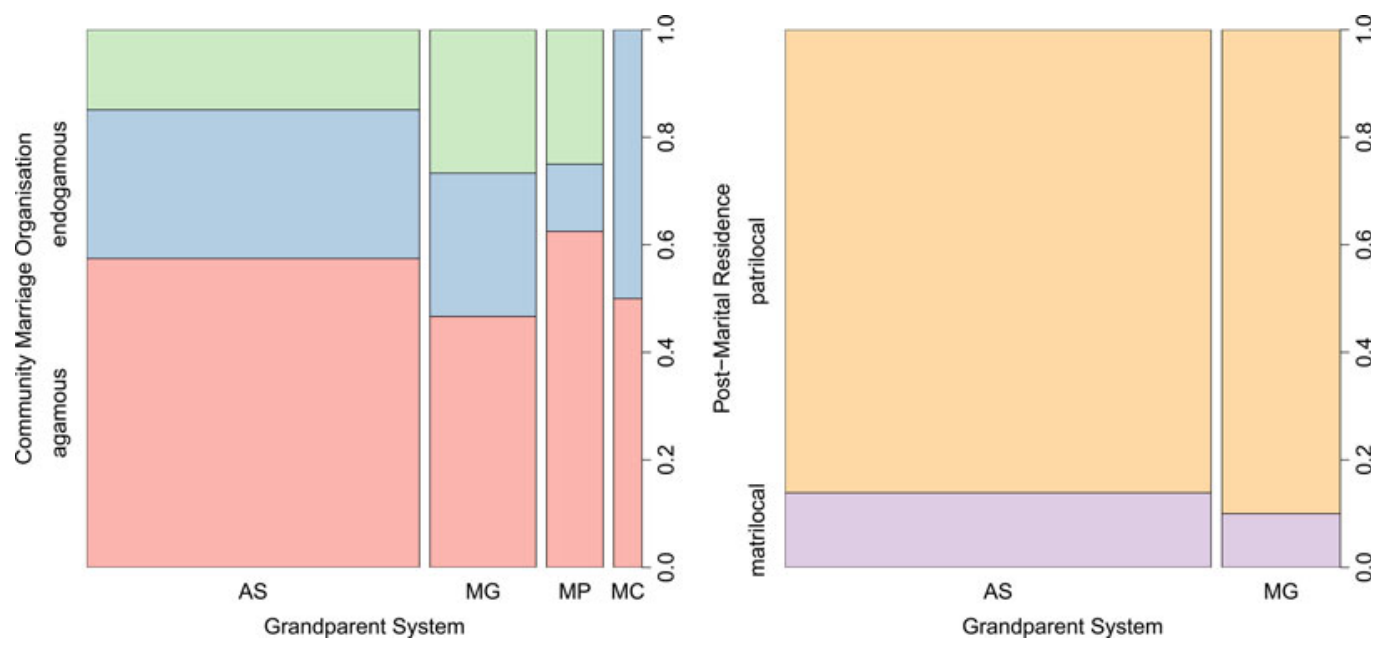

Figure 4. The distribution of Pama-Nyungan grandparent systems by social norms. AS, all separate; $M G$, merged genders (MM = FM and $\mathrm{MF}=\mathrm{FF})$; $\mathrm{MP}$, merged parallel $(\mathrm{MM}=\mathrm{FF})$; and $\mathrm{MC}$, merged cross $(\mathrm{FM}=\mathrm{MF})$. The differences shown are not statistically significant.

There was no apparent relationship between the four most frequent grandparental systems and whether the cross-cousin systems merge by gender (Fisher's exact test, $P=0.672$ ), merge by lineage (Fisher's exact test, $P=0.140$ ), or have four separate terms (Fisher's exact test, $P=0.704$ ). A generic cousin term was common in AS $(79 \%)$ or MG $(100 \%)$ systems and rare in MC $(50 \%)$ or MP (38\%) systems (Fisher's exact test, $P=0.022$ ), but this difference did not survive phylogenetic correction $(\mathrm{pMCMC}=0.220)$. 


\section{Discussion}

Here we have shown that, contrary to previous expectations, shifts in Pama-Nyungan grandparent systems do not correspond to changes in community marriage organisation or social norms. Furthermore, although some aspects of grandparental systems seem to change in concert with shifts in grandchild and cross-cousin terms, many of these patterns do not survive phylogenetic correction. Thus, we find no strong evidence of a single set of kinship typologies within Pama-Nyungan, especially one such as those based on cousin terminologies in the sense of Murdock (1949).

We find 10 grandparent systems, out of the 15 combinatorial possibilities, across 134 languages, with most languages falling into a single category (four separate terms, 61\%). Given the high phylogenetic signal in the data, some of this under-dispersion could be due to phylogenetic conservatism; grandparent systems could be slow to change. Alternatively, although we find no correlation between grandparent system and the two social variables tested here (community marriage organisation and post-marital residence), grandparent systems could be adaptively flexible to other social, cultural or cognitive drivers. Furthermore, the fact that we find no evidence of spatial correlation could suggest that grandparental systems are not strongly shaped by any drivers that themselves have strong spatial signals (such as an environmental variable) or that kinship systems are not horizontally diffused to nearby populations at any meaningful rate. It is unclear, however, how much these patterns may translate to other groups. For example, an analysis of Khoisan sibling terminology found much less evidence of phylogenetic conservatism within the kinship system (Boden et al., 2014), while the strong support for reconstructed states for most nodes in an analysis of Austronesian sibling terms suggests a strong phylogenetic signature (Jordan, 2011), indicating that regional-level processes may be overriding any universal rules regarding kinship system change. A potential cause of this may be the imprecision of straight-line distance as a proxy for inter-group contact, given the roles geographical features (such as navigable waterways or impassable mountains) or cultural practices can play in facilitating horizontal transmission (Bowern, 2013). Within the Australian context specifically, we here only study Pama-Nyungan languages and thus cannot rule out borrowing from non-Pama-Nyungan languages, particularly in Arnhem Land (Bouckaert et al., 2018; Bowern \& Atkinson, 2012; Heath, 1978). It is also important to emphasise the distinction between changes in overall systems (the subject of this study) and changes in terms (upon which we do not remark), as preliminary analyses of PamaNyungan sibling terms indicate that lexical items themselves change more frequently than systems (Bowern, 2014).

Furthermore, we find that grandparent systems do not change randomly. Among the four most common systems, we do not observe transitions between all states; instead, the ancestral state of four separate terms can shift to either merged parallel grandparents $(\mathrm{MM}=\mathrm{FF})$ or, more rarely, to merged genders $(\mathrm{MM}=\mathrm{FM}$ and $\mathrm{MF}=\mathrm{FF})$, either of which secondary states can subsequently shift to merged cross grandparents $(\mathrm{MF}=\mathrm{FM})$. Intriguingly, these shifts appear to occur without evidence of a stable, long-term intermediate stage (for example, from merged parallel to merged cross without either a system where $\mathrm{MM}=\mathrm{FF}$ and $\mathrm{MF}=\mathrm{FM}$ or by returning back to four separate terms). A similar phenomenon can be observed in examining the history of the terms themselves. For example, many Pama-Nyungan languages have a term ${ }^{\star} k a m i$ that means MM, while other languages have this term as FM, with no strong evidence of an intermediate stage where ${ }^{\star} k a m i$ means both MM and FM (McConvell, 2013).

Although kinship systems are generally approached from anthropological or linguistic perspectives, they also present a combinatorial puzzle, with important implications for the study of kinship typologies. Classifications of family members can be abstractly conceptualised as partitions of a set, meaning that the number of possible systems for a family of $n$ roles corresponds to the $n$th Bell number. Thus, four grandparents, with no mechanism for distinguishing speaker gender, have 15 possible classification systems; while a system with 16 roles (say, cousins that distinguish lineage, referent gender, and speaker gender) has 10,480,142,147 possible classifications. Obviously, not all possible systems are found in the world's languages; if nothing else, there are only approximately 7,000 languages spoken today (Anderson, 2010). Some of this variation is thought to be structured by basic cognitive constraints; 
Kemp and Regier (2012), for example, surveyed 487 world languages and found 410 different kinship systems that nearly all adhered to a single-dimensional trade-off between simplicity and informativeness. Other variation is thought to be shaped by social structure. For example, Rácz et al. (2019) found that Murdock's cousin typologies correlate with a society's rules for marriage and descent in a sample of 936 languages (none Australian). Within the Australian context, so-called Omaha skewing, or merging terms across generations within a system with separate words for patrilineal cross-cousins, is thought to be related to exogamy to avoid demographic collapse in small population groups (McConvell, 2012; McConvell \& Dousset, 2012). On the other hand, Guillon and Mace (2016) find no relationship between Bantu cousin systems and either descent or residence norms, suggesting that not all language-culture coevolution hypotheses with microevolutionary support will hold true at the macroevolutionary level.

Many studies in cross-cultural human kinship attempt to classify the vast variation into a small number of socially meaningful typologies (Dzibel \& Dziebel, 2007; Fox, 2008; Morgan, 1871; Murdock, 1949; Read, 2013). Our results suggest that grandparent and cross-cousin systems are uncorrelated, and thus that a Murdock-style classification based only on cross-cousins will obscure variation in other aspects of kinship. Furthermore, many of our correlations between grandparental systems and grandchild systems are only statistically significant when the autocorrelation owing to shared cultural history is ignored (see supplementary material), indicating that observations of kin term synchrony uncorrected for phylogeny may be invalid. Studies of Australian kinship have often discarded the globally based six-category Murdock system in favour of systems that are more meaningful for the continent (McConvell \& Hendery, 2017); by studying systematic variation at the level of the term within a phylogenetic context, we are able to go even further and suggest that kin term synchrony, and thus stable kinship typologies, may be rarer than previously thought.

By focusing on a large expansion of hunter-gatherer languages, we are able to complement previous phylogenetic studies of kinship systems in agricultural societies and provide additional data in service of testing regularities in cultural evolution. Humans lived as hunter-gathers for the vast majority of our species' history, and hunter-gatherer kinship is thought to differ from that of agriculturalists in terms of residence patterns (K. R. Hill et al., 2011), inbreeding rates (Walker \& Bailey, 2014) and the speed at which systems can change (Opie et al., 2014). Hunter-gather languages are also generally assumed to have a high level of borrowing (Dixon, 1998; Nettle, 1999), although recent work has demonstrated that loan rates in hunter-gatherer languages from Australia, Amazonia and western North America are broadly comparable with, albeit more variable than, those in neighbouring agricultural languages (Bowern et al., 2011). We find no evidence of spatial autocorrelation in our data, and thus no particular evidence of borrowing. Instead, we find a strong phylogenetic signal and high phylogenetic conservatism, indicating that grandparental systems are largely inherited from previous generations and neither change flexibly nor are commonly borrowed.

In summary, we found no evidence that Pama-Nyungan grandparent systems are related to postmarital residence or community marriage organisation. Most languages in our survey had a system with four separate terms, which occasionally shifted to mergers along either referent gender or the parallel grandparents, with a few languages subsequently shifting to a cross merger. We found no evidence that these shifts in grandparent terms relate to shifts in systems for classifying cross-cousins, although we do find moderate evidence linking grandparental and grandchild terms, indicating that care is needed in the assumption of a small number of kinship typologies, particularly in studies lacking phylogenetic correction.

Acknowledgements. We thank Sam Passmore for help with data curation and Alexandra Marley and three anonymous reviewers for comments on a previous version of this manuscript.

Financial support. CS and FMJ were supported by European Research Council Starting Grant no. 639291 VARIKIN (to FMJ). CB was supported by NSF grants BCS-0844550 and BCS-1423711.

Author contributions. CS, FMJ and CB designed research; RD, CB and CS collected and interpreted data; CS performed analyses; CS wrote the paper, with editing from all authors. 
Conflict of interest. None.

Data availability statement. The data behind this paper can be found at https://doi.org/10.5281/zenodo.3832215. Full data sources can be found in the supplementary material.

Supplementary material. To view supplementary material for this article, please visit https://doi.org/10.1017/ehs.2020.31.

\section{References}

Anderson, S. R. (2010). How many languages are there in the world. Linguistic Society of America.

Atkinson, Q. D., \& Gray, R. D. (2005). Curious parallels and curious connections - Phylogenetic thinking in biology and historical linguistics. Systematic Biology, 54(4), 513-526. doi:10.1080/10635150590950317

Binford, L. R. (2001). Constructing frames of reference: An analytical method for archaeological theory building using ethnographic and environmental data sets (1st ed.). University of California Press.

Birchall, J., \& Jordan, F. M. (2019). Dossier 'New perspectives on kinship terminology in Tupian and Cariban languages'. Boletim do Museu Paraense Emílio Goeldi. Ciências Humanas, 14(1), 11-14. doi:10.1590/1981.81222019000100002

Blute, M., \& Jordan, F. M. (2018). The evolutionary approach to history. Oxford handbook of evolution, biology, and society. Oxford University Press. doi:10.1093/oxfordhb/9780190299323.013.32

Boden, G., Güldemann, T., \& Jordan, F. (2014). Khoisan sibling terminologies in historical perspective: A combined anthropological, linguistic and phylogenetic comparative approach. In T. Güldemann, \& A-M. Fehn (Eds.), Beyond 'Khoisan': Historical relations in the Kalahari Basin (pp. 67-100). (Current Issues in Linguistic Theory; Vol. 330). John Benjamins Publishing Company. doi:10.1075/cilt.330.03bod

Bouckaert, R. R., Bowern, C., \& Atkinson, Q. D. (2018). The origin and expansion of Pama-Nyungan languages across Australia. Nature Ecology \& Evolution, 2(4), 741. doi:10.1038/s41559-018-0489-3

Bowern, C. (2010). Historical linguistics in Australia: Trees, networks and their implications. Philosophical Transactions of the Royal Society B: Biological Sciences, 365(1559), 3845-3854. doi:10.1098/rstb.2010.0013

Bowern, C. (2013). Relatedness as a factor in language contact. Journal of Language Contact, 6(2), 411-432. doi:10.1163/ 19552629-00602010

Bowern, C. (2014). Lexical stability and kinship patterns in Australian languages. Paper presented at the Linguistic Society pf America, Minneapolis, MN. Retrieved from pamanyungan.sites.yale.edu/kinship

Bowern, C. (2016). Chirila: contemporary and historical resources for the indigenous languages of Australia. Language Documentation, 10, 44.

Bowern, C., \& Atkinson, Q. (2012). Computational phylogenetics and the internal structure of Pama-Nyungan. Language, 88 (4), 817-845. doi:10.1353/lan.2012.0081

Bowern, C., Epps, P., Gray, R., Hill, J., Hunley, K., McConvell, P., \& Zentz, J. (2011). Does lateral transmission obscure inheritance in hunter-gatherer languages? PLOS ONE, 6(9), e25195. doi:10.1371/journal.pone.0025195

Bramanti, B., Thomas, M. G., Haak, W., Unterlaender, M., Jores, P., Tambets, K., ... Burger, J. (2009). Genetic discontinuity between local hunter-gatherers and central Europe's first farmers. Science, 326(5949), 137-140. doi:10.1126/ science.1176869

Chagnon, N. A., Lynch, R. F., Shenk, M. K., Hames, R., \& Flinn, M. V. (2017). Cross-cousin marriage among the Yanomamö shows evidence of parent-offspring conflict and mate competition between brothers. Proceedings of the National Academy of Sciences, 114(13), E2590-E2607. doi:10.1073/pnas.1618655114

Chapais, B. (2014). Complex kinship patterns as evolutionary constructions, and the origins of sociocultural universals. Current Anthropology, 55(6), 751-783. doi:10.1086/678972

Clendon, M. (2006). Reassessing Australia's linguistic prehistory. Current Anthropology, 47(1), 39-61. doi:10.1086/497671

Creanza, N., Kolodny, O., \& Feldman, M. W. (2017). Cultural evolutionary theory: How culture evolves and why it matters. Proceedings of the National Academy of Sciences of the United States of America. doi:10.1073/pnas.1620732114

Currie, T. E., Greenhill, S. J., \& Mace, R. (2010). Is horizontal transmission really a problem for phylogenetic comparative methods? A simulation study using continuous cultural traits. Philosophical Transactions of the Royal Society B: Biological Sciences, 365(1559), 3903-3912. doi:10.1098/rstb.2010.0014

da Silva, S. G., \& Tehrani, J. J. (2016). Comparative phylogenetic analyses uncover the ancient roots of Indo-European folktales. Royal Society Open Science, 3(1), 150645. doi:10.1098/rsos.150645

Dixon, R. M. W. (1990). The origin of 'mother-in-law vocabulary' in two Australian languages. Anthropological Linguistics, 32 $(1 / 2), 1-56$

Dixon, R. M. W. (1998). The rise and fall of languages. Cambridge University Press.

Dole, G. E. (1969). Generation kinship nomenclature as an adaptation to endogamy. Southwestern Journal of Anthropology, 25(2), 105-123.

Dray, S., \& Dufour, A.-B. (2007). The ade4 package: Implementing the duality diagram for ecologists. Journal of Statistical Software, 22(1), 1-20. doi:10.18637/jss.v022.i04 
Dunn, M., Greenhill, S. J., Levinson, S. C., \& Gray, R. D. (2011). Evolved structure of language shows lineage-specific trends in word-order universals. Nature, 473(7345), 79-82. doi:10.1038/nature09923

Dzibel, G. V., \& Dziebel, G. V. (2007). The genius of kinship: The phenomenon of kinship and the global diversity of kinship terminologies. Cambria Press.

Fortunato, L., \& Jordan, F. (2010). Your place or mine? A phylogenetic comparative analysis of marital residence in Indo-European and Austronesian societies. Philosophical Transactions of the Royal Society B: Biological Sciences, 365 (1559), 3913-3922. doi:10.1098/rstb.2010.0017

Fox, R. (2008). Kinship and marriage: An anthropological perspective. Cambridge University Press.

Godelier, M. (2012). The metamorphoses of kinship. Verso.

Godelier, M., Trautmann, T. R., \& Tjon Sie Fat, F. E. (1998). Transformations of kinship, M. Godelier, T. R. Trautmann, \& F. E. T. S. Fat (Eds.). Smithsonian Books.

Goody, J. (1970). Cousin terms. Southwestern Journal of Anthropology, 26(2), 125-142.

Gray, R. D., Greenhill, S. J., \& Ross, R. M. (2007). The pleasures and perils of Darwinizing culture (with phylogenies). Biological Theory, 2(4), 360-375. doi:10.1162/biot.2007.2.4.360

Guillon, M., \& Mace, R. (2016). A phylogenetic comparative study of Bantu kinship terminology finds limited support for its co-evolution with social organisation. PLOS ONE, 11(3), e0147920. doi:10.1371/journal.pone.0147920

Hadfield, J. D. (2010). MCMC methods for multi-response generalized linear mixed models: The MCMCglmm R package. Journal of Statistical Software, 33(2), 1-22. doi:10.18637/jss.v033.i02

Hammarström, H., Bank, S., Forkel, R., \& Haspelmath, M. (2018). Glottolog 3.2. Max Planck Institute for the Science of Human History. Retrieved from http://glottolog.org

Harmon, L. J., Weir, J. T., Brock, C. D., Glor, R. E., \& Challenger, W. (2008). GEIGER: Investigating evolutionary radiations. Bioinformatics, 24(1), 129-131. doi:10.1093/bioinformatics/btm538

Harvey, P. H., \& Pagel, M. (1991). The comparative method in evolutionary biology. Oxford University Press.

Haspelmath, M. (2008). Loanword typology: Steps toward a systematic crosslinguistic study of lexical borrowability. In: T. Stolz, D. Bakker \& R. Salas Palomo (Eds.), Aspects of Language Contact: New Theoretical, Methodological and Empirical Findings with Special Focus on Romancisation Processes (pp. 43-62). Berlin: Mouton de Gruyter.

Haspelmath, M., König, E., Oesterreicher, W., \& Raible, W. (2001). Language typology and language universals: An international handbook. Walter de Gruyter.

Hawkes, K., O’Connell, J. F., Jones, N. G. B., Alvarez, H., \& Charnov, E. L. (1998). Grandmothering, menopause, and the evolution of human life histories. Proceedings of the National Academy of Sciences, 95(3), 1336-1339. doi:10.1073/ pnas.95.3.1336

Heath, J. (1978). Linguistic diffusion in Arnhem Land. Australian Institute of Aboriginal Studies.

Hill, J. H. (1978). Language contact systems and human adaptations. Journal of Anthropological Research, 34(1), 1-26. doi:10.1086/jar.34.1.3629628

Hill, K., \& Hurtado, A. M. (1991). The evolution of premature reproductive senescence and menopause in human females. Human Nature, 2(4), 313-350. doi:10.1007/BF02692196

Hill, K. R., Walker, R. S., Božičević, M., Eder, J., Headland, T., Hewlett, B., ... Wood, B. (2011). Co-residence patterns in hunter-gatherer societies show unique human social structure. Science, 331(6022), 1286-1289. doi:10.1126/ science. 1199071

Hrdy, S. B. (2011). Mothers and Others: The Evolutionary Origins of Mutual Understanding. Harvard University Press.

Jordan, F. (2011). A phylogenetic analysis of the evolution of Austronesian sibling terminologies. Human Biology, 83(2), 297-321.

Keesing, R. M. (1975). Kin groups and social structure. Holt, Rinehart and Winston.

Kemp, C., \& Regier, T. (2012). Kinship categories across languages reflect general communicative principles. Science, 336 (6084), 1049-1054. doi:10.1126/science.1218811

Kirby, K. R., Gray, R. D., Greenhill, S. J., Jordan, F. M., Gomes-Ng, S., Bibiko, H.-J., .. Gavin, M. C. (2016). D-PLACE: A global database of cultural, linguistic and environmental diversity. PLOS ONE, 11(7), e0158391. doi:10.1371/ journal.pone.0158391

Kroeber, A. L. (1909). Classificatory systems of relationship. The Journal of the Royal Anthropological Institute of Great Britain and Ireland, 39, 77-84. doi:10.2307/2843284

Lacrampe, S., McConvell, P., \& Kelly, P. (2018). Skin, kin and clan. ANU Press.

Mace, R., \& Holden, C. J. (2005). A phylogenetic approach to cultural evolution. Trends in Ecology \& Evolution, 20(3), 116121. doi:10.1016/j.tree.2004.12.002

Mace, R., Pagel, M., Bowen, J. R., Gupta, B. K. D., Otterbein, K. F., Ridley, M., ... Voland, E. (1994). The comparative method in anthropology. Current Anthropology, 35(5), 549-564.

Malaspinas, A.-S., Westaway, M. C., Muller, C., Sousa, V. C., Lao, O., Alves, I., ... Willerslev, E. (2016). A genomic history of Aboriginal Australia. Nature, 538(7624), 207-214. doi:10.1038/nature18299

McConvell, P. (2012). Omaha skewing in Australia: Overlays, dynamism, and change. In T. R Trautmann \& P. M. Whiteley (Eds.), Crow-Omaha: New light on a classic problem of kinship analysis (pp. 243-260). Tucson, AZ: University of Arizona Press. 
McConvell, P. (2013). Granny got cross: Semantic change of kami 'mother's mother' to 'father's mother' in Pama-Nyungan. In R. Mailhammer (Ed.), Lexical and Structural Etymology: Beyond Word Histories (pp. 147-184). De Gruyter Mouton.

McConvell, P., \& Bowern, C. (2011). The prehistory and internal relationships of Australian languages. Language and Linguistics Compass, 5(1), 19-32. doi:10.1111/j.1749-818X.2010.00257.x

McConvell, P., \& Dousset, L. (2012). Tracking the Dynamics of Kinship and Social Category Terms with AustKin II. 3th Conference of the European Chapter of the Association for Computational Linguistics (EACL2012), Apr 2012, Avignon, France. pp. 98107.

McConvell, P., \& Hendery, R. (2017). What is 'Kariera'? Detecting systems and overlap in Australian kinship using the AustKin database. Oceania, 87(2), 188-208. doi:10.1002/ocea.5155

McConvell, P., Keen, I., \& Hendery, R. (2013). Kinship systems: Change and reconstruction (1st ed.). University of Utah Press.

Møllegaard, S., \& Jæger, M. M. (2015). The effect of grandparents' economic, cultural, and social capital on grandchildren's educational success. Research in Social Stratification and Mobility, 42, 11-19. doi:10.1016/j.rssm.2015.06.004

Moravec, J. C., Atkinson, Q., Bowern, C., Greenhill, S. J., Jordan, F. M., Ross, R. M., .. Cox, M. P. (2018). Post-marital residence patterns show lineage-specific evolution. Evolution and Human Behavior, 39(6), 594-601. doi:10.1016/ j.evolhumbehav.2018.06.002

Morgan, L. H. (1871). Systems of consanguinity and affinity of the human family (Vol. 218). Smithsonian Institution.

Murdock, G. P. (1949). Social structure. Macmillan.

Murdock, G. P. (1967). Ethnographic atlas: A summary. Ethnology, 6, 109-236.

Murdock, G. P. (1968). Patterns of sibling terminology. Ethnology, 7(1), 1-24. doi:10.2307/3772805

Nerlove, S., \& Romney, A. K. (1967). Sibling terminology and cross-sex behavior. American Anthropologist, 69(2), $179-187$. doi:10.1525/aa.1967.69.2.02a00050

Nettle, D. (1999). Linguistic diversity. Oxford University Press.

Normile, D. (2017). China's childhood experiment. Science, 357(6357), 1226-1230. doi:10.1126/science.357.6357.1226

Nunn, C. L., Mulder, M. B., \& Langley, S. (2006). Comparative methods for studying cultural trait evolution: a simulation study. Cross-cultural Research, 40(2), 177-209. doi:10.1177/1069397105283401

Opie, C., Shultz, S., Atkinson, Q. D., Currie, T., \& Mace, R. (2014). Phylogenetic reconstruction of Bantu kinship challenges Main Sequence Theory of human social evolution. Proceedings of the National Academy of Sciences, 111(49), 17414-17419. doi:10.1073/pnas.1415744111

Pagel, M. (1994). Detecting correlated evolution on phylogenies: a general method for the comparative analysis of discrete characters. Proceedings of the Royal Society of London. Series B: Biological Sciences, 255(1342), 37-45. doi:10.1098/ rspb.1994.0006

Pagel, M., Meade, A., \& Barker, D. (2004). Bayesian estimation of ancestral character states on phylogenies. Systematic Biology, 53(5), 673-684. doi:10.1080/10635150490522232

Paradis, E., Claude, J., \& Strimmer, K. (2004). APE: Analyses of phylogenetics and evolution in R language. Bioinformatics, 20 (2), 289-290. doi:10.1093/bioinformatics/btg412

Parkin, R. (1997). Kinship: An introduction to the basic concepts. Wiley-Blackwell.

Parkin, R. (2012). Kinship as classification: Towards a paradigm of change. Journal of the Anthropological Society of Oxford, 4 (2), 183-211.

Pashos, A. (2017). Asymmetric caregiving by grandparents, aunts, and uncles and the theories of kin selection and paternity certainty: How does evolution explain human behavior toward close relatives? Cross-cultural Research, 51(3), $263-284$. doi:10.1177/1069397117697671

Perry, G., \& Daly, M. (2017). A model explaining the matrilateral bias in alloparental investment. Proceedings of the National Academy of Sciences, 114(35), 9290-9295. doi:10.1073/pnas.1705910114

Powell, A., Shennan, S., \& Thomas, M. G. (2009). Late Pleistocene demography and the appearance of modern human behavior. Science, 324(5932), 1298-1301. doi:10.1126/science.1170165

Rácz, P., Passmore, S., \& Jordan, F. M. (2019). Social practice and shared history, not social scale, structure cross-cultural complexity in kinship systems. Topics in Cognitive Science, 12(2), 1-22. doi:10.1111/tops.12430.

Read, D. W. (2013). A new approach to forming a typology of kinship terminology systems: From Morgan and Murdock to the present. Structure and Dynamics, 6(1), 1-26.

Reiss, P. J. (1962). The extended kinship system: Correlates of and attitudes on frequency of interaction. Marriage and Family Living, 24(4), 333-351. doi:10.2307/349102

Rivers, W. H. R. (1914). Kinship and social organisation. Constable.

Scheffler, H. W. (1978). Australian kin classification. Cambridge University Press.

Schulz, J. F., Bahrami-Rad, D., Beauchamp, J. P., \& Henrich, J. (2019). The Church, intensive kinship, and global psychological variation. Science, 366(6466). doi:10.1126/science.aau5141

Sear, R., \& Mace, R. (2008). Who keeps children alive? A review of the effects of kin on child survival. Evolution and Human Behavior, 29(1), 1-18. doi:10.1016/j.evolhumbehav.2007.10.001

Sear, R., Mace, R., \& McGregor, I. A. (2000). Maternal grandmothers improve nutritional status and survival of children in rural Gambia. Proceedings: Biological Sciences, 267(1453), 1641-1647. 
Sheehan, O., Watts, J., Gray, R. D., \& Atkinson, Q. D. (2018). Coevolution of landesque capital intensive agriculture and sociopolitical hierarchy. Proceedings of the National Academy of Sciences, 115(14), 3628-3633. doi:10.1073/ pnas. 1714558115

Surowiec, A., Snyder, K. T., \& Creanza, N. (2019). A worldwide view of matriliny: Using cross-cultural analyses to shed light on human kinship systems. Philosophical Transactions of the Royal Society B: Biological Sciences, 374(1780), 20180077. doi:10.1098/rstb.2018.0077

Tylor, E. B. (1889). On a method of investigating the development of institutions; Applied to laws of marriage and descent. The Journal of the Anthropological Institute of Great Britain and Ireland, 18, 2450272.

Walker, R. S., \& Bailey, D. H. (2014). Marrying kin in small-scale societies. American Journal of Human Biology, 26(3), 384388. doi:10.1002/ajhb.22527

White, I. (1981). Generation moieties in Australia: Structural, social and ritual implications. Oceania, 52(1), 6-27. doi:10.1002/j.1834-4461.1981.tb01482.x

Whitehouse, H., François, P., Savage, P. E., Currie, T. E., Feeney, K. C., Cioni, E., ... Turchin, P. (2019). Complex societies precede moralizing gods throughout world history. Nature, 568(7751), 226. doi:10.1038/s41586-019-1043-4

Woodward, J. C. (1978). All in the family: Kinship lexicalization across sign languages. Sign Language Studies, 19, $121-138$. Yallop, C. (1977). Alyawarra, an Aboriginal language of central Australia. Australian Institute of Aboriginal Studies.

Cite this article: Sheard C, Bowern C, Dockum R, Jordan FM (2020). Pama-Nyungan grandparent systems change with grandchildren, but not cross-cousin terms or social norms. Evolutionary Human Sciences 2, e30, 1-16. https://doi.org/ 10.1017/ehs.2020.31 